\title{
Fractura de espina tibial bilateral en adulto
}

\author{
Bilateral tibial spine fracture in an adult \\ Fernando Chico Carpizo, * Edgar Turrubiates Lucero, * Luis Gerardo Domínguez Gasca, ${ }^{\ddagger}$ \\ Andrew Carlomaría Daniel López Márquez, ${ }^{*}$ Luis Gerardo Domínguez Carrillo ${ }^{\S}$ \\ Citar como: Chico CF, Turrubiates LE, Domínguez GLG, López MACD, Domínguez CLG. Fractura de espina \\ tibial bilateral en adulto. Acta Med GA. 2022; 20 (1): 71-73. https://dx.doi.org/10.35366/103560
}

\section{Resumen}

Introducción: La fractura de espina tibial es poco frecuente, con incidencia de $3 / 100,000$, su presencia bilateral es extraordinaria. Caso clínico: Mujer de 30 años que sufrió caída de $1.5 \mathrm{~m}$ de altura, colisionando el piso con talones que originó carga axial, hiperextensión y valgo forzado de ambas rodillas, sus radiografías y tomografía mostraron fractura de espina tibial bilateral, recibió tratamiento mediante artroscopia, efectuando reducción y fijación con suturas de alta resistencia y alambres de Kirschner. Conclusiones: El manejo artroscópico de este tipo de lesiones brinda resultados funcionales y disminuyen la comorbilidad, evitando una segunda cirugía para retiro de material.

Palabras clave: Fractura de espina tibial, artroscopia, fijación, sutura de alta resistencia, clavos Kirschner.

\section{INTRODUCCIÓN}

La fractura de espina tibial es poco frecuente, con incidencia de 3/100,000. Habitualmente son fracturas secundarias a mecanismo de hiperextensión de la rodilla y valgo o rotación externa forzada, por lo general se presentan en pacientes pediátricos, con incremento de incidencia en adultos, por aumento en actividades deportivas y en especial asociadas con accidentes automovilísticos. ${ }^{1} \mathrm{Al}$ presentarse en la institución una paciente adulta con fractura de

\section{Abstract}

Introduction: Tibial spine fracture is rare, with an incidence of $3 / 100,000$; bilateral fractures are of extraordinary presentation. Case report: 30-year-old female who suffered a fall of 1.5 $\mathrm{m}$ height colliding the floor with heels that caused axial load, hyperextension, and forced valgus of both knees, her $\mathrm{X}$-rays and tomography showed bilateral tibial spine fracture, he received arthroscopic treatment, carrying out reduction and fixation with high-strength sutures and Kirschner wires. Conclusions: Arthroscopic management of this type of injury provides functional results and reduces comorbidity, avoiding a second surgery to remove material.

Keywords: Tibial spine fracture, arthroscopy, fixation, high strength sutures, Kirschner wires.

* Ortopedista, Cirugía Articular. División de Cirugía del Hospital General Regional de León. León, Guanajuato, México.

₹ Ortopedista, Cirugía Articular. División de Cirugía del Hospital Angeles León. León, Guanajuato, México.

§ Especialista en Medicina de Rehabilitación. Catedrático de la Facultad de Medicina de León, Universidad de Guanajuato. México.

espina tibial bilateral, siendo una situación extraordinaria, pues solamente existe un reporte en la literatura de un caso pediátrico, se realizó el presente reporte. ${ }^{1}$

\section{CASO CLÍNICO}

Mujer de 30 años, profesionista, sin antecedentes de importancia, que acude tras caída de 1.5 metros de altura, colisionando ambos talones contra el piso, lo que originó carga axial, hiperextensión y valgo forzado de ambas ro-

www.medigraphic.com/actamedica 

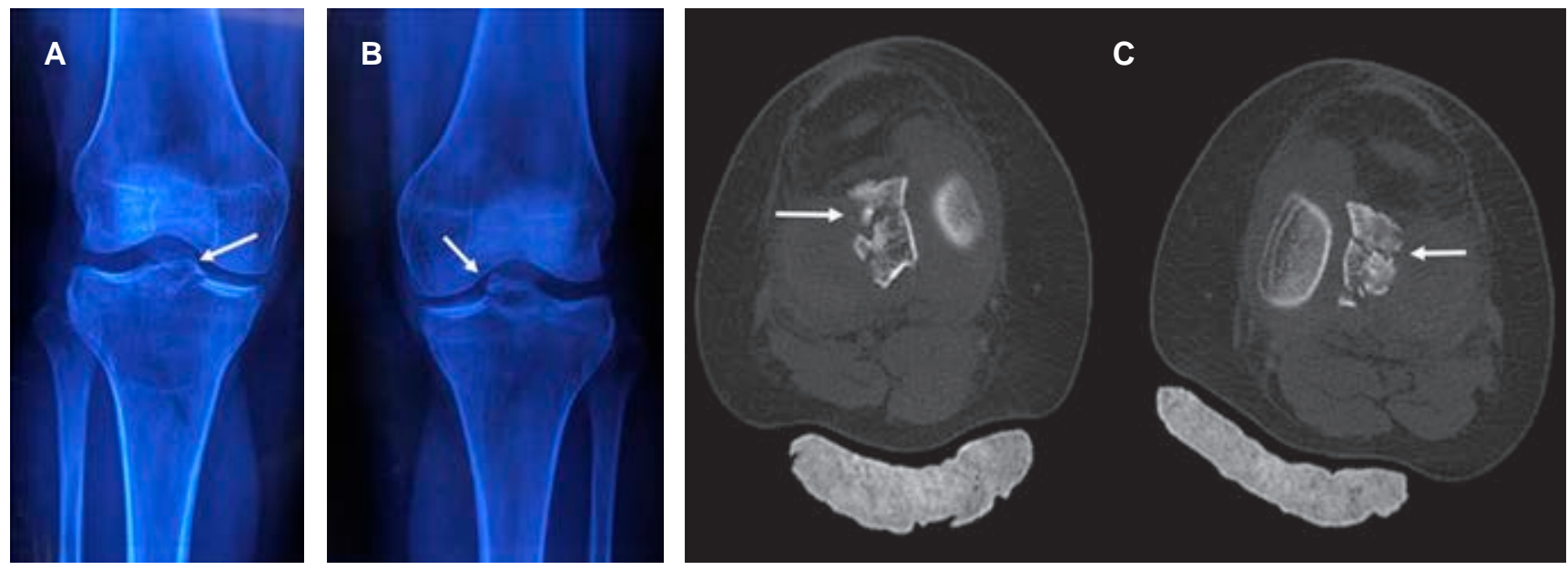

Figura 1: A, B) Radiografías anteroposteriores de ambas rodillas, mostrando fractura de espina tibial bilateral (flechas). C) Imagen de tomografía computarizada de rodillas, en corte axial, mostrando trazos de fractura de espina tibial bilateral (flechas).
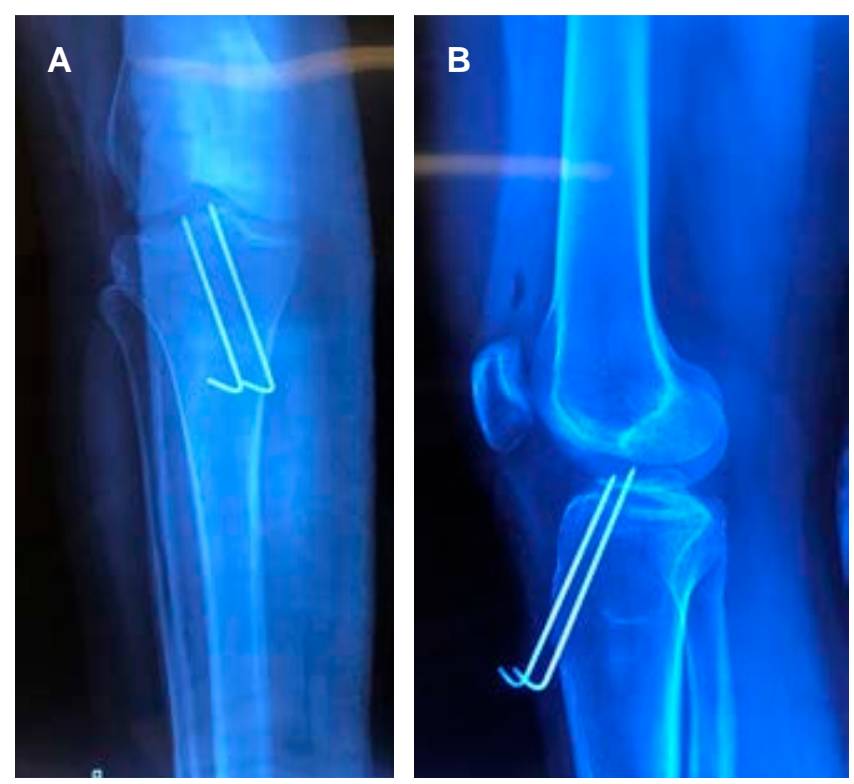

Figura 2: Radiografías simples anteroposterior (A) y lateral (B) de rodilla derecha, mostrando clavillos de Kirschner para fijar fractura de espina tibial.

dillas, presentando dolor inmediato e imposibilidad para la ambulación. A la exploración gran edema de ambas rodillas por hemartrosis, imposibilidad por dolor para efectuar movimientos de flexoextensión. Resto de exploración normal. Se efectúan radiografías simples de ambas rodillas, encontrando fractura de la espina tibial bilateral (Figura $1 \mathrm{~A}$ y B), con discreto desplazamiento. Se efectúa tomografía computarizada observando al menos cuatro fragmentos y trazos de fractura (Figura 1C), correspondiendo fractura de espina tibial anterior izquierda Meyers y McKeever tipo IIIB y fractura de espina tibial anterior derecha, Meyers y McKeever tipo II. Se realiza tratamiento quirúrgico mediante artroscopia con guía para ligamento cruzado anterior, reducción y fijación con sutura de alta resistencia tipo FiberWire 2.0 y alambres de Kirschner No. 2.0 (Figura 2) en rodilla izquierda y derecha respectivamente, derivándose a rehabilitación a las tres semanas, se retiraron los alambres de Kirschner a las seis semanas en consultorio; la evolución fue satisfactoria logrando a cuatro meses de rehabilitación: marcha independiente, arcos de movimiento de $130^{\circ}$ en ambas rodillas, con fuerza muscular 5/5 en escala de Daniels y pruebas de cajón anterior y Lachman negativas.

\section{DISCUSIÓN}

La fractura de espina tibial es poco frecuente, existe predominio en el género masculino con una relación de 4:1 en adultos, principalmente en rodilla izquierda. ${ }^{1}$ En relación con la presentación bilateral, sólo existe el reporte Andersen y Mejdahl ${ }^{2}$ en 1993 del caso de una niña de ocho años.

La artroscopia es el método de elección para el manejo de las fracturas de espina tibial, reduciendo las complicaciones de tejidos blandos, dolor postoperatorio, estancia hospitalaria y evitando el retraso en la rehabilitación, ${ }^{3}$ ya que permite una visualización intraarticular completa, reducción precisa de los fragmentos de la fractura, tratamiento de lesiones asociadas de tejidos blandos y la extracción de fragmentos libres. ${ }^{4}$ Existen numerosas técnicas de fijación, aunque aún no hay una guía específica de tratamiento.

El manejo artroscópico ha demostrado una recuperación de la flexoextensión completa aproximadamente a los cinco 
a seis meses en más de 95\% de los pacientes; el tratamiento realizado a los 15 días posteriores a la lesión representa un riesgo significativo para la rigidez articular, por lo que es de gran importancia su diagnóstico y manejo oportuno.

Estudios comparativos de los diferentes métodos de fijación no han establecido diferencia en los resultados radiográficos y clínicos; además de la ventaja que brindan el uso de suturas de alta resistencia y clavos Kirschner al evitar una segunda cirugía para retirar el material de fijación. ${ }^{5}$

Existen estudios biomecánicos en cadáver, donde se ha comprobado que la fijación con suturas transóseas brindan resultados más confiables que la fijación con tornillos canulados, ${ }^{6}$ pudiendo iniciar inmediatamente con movimiento y apoyo completo de la extremidad afectada. La desventaja es la mayor dificultad técnica y mayor tiempo quirúrgico.

\section{CONCLUSIONES}

El manejo artroscópico de la fractura de espina tibial, utilizando suturas de alta resistencia con apoyo de clavos Kirschner, brinda resultados funcionales y disminuye la comorbilidad, evitando una segunda cirugía para retiro de material.

\section{REFERENCIAS}

1. Strauss EJ, Kaplan DJ, Weinberg ME, Egol J, Jazrawi LM. Arthroscopic management of tibial spine avulsion fractures: principles and techniques. J Am Acad Orthop Surg. 2018; 26 (10): 360-367. doi: 10.5435/JAAOS-D-16-00117.

2. Andersen JW, Mejdahl S. Bilateral fracture of the tibial spine. Acta Orthop Belg. 1993; 59 (4): 394-397.

3. Osti L, Buda M, Soldati F, Del Buono A, Osti R, Maffulli N. Arthroscopic treatment of tibial eminence fracture: a systematic review of different fixation methods. Br Med Bull. 2016; 118 (1): 73-90.

4. Senekovic $V$, Balazic M. Bioabsorbable sutures versus screw fixation of displaced tibial eminence fractures: a biomechanical study. Eur J Orthop Surg Traumatol. 2014; 24 (2): 209-216. doi: 10.1007/ s00590-013-1176-3.

5. Bong MR, Romero A, Kubiak E, lesaka K, Heywood CS, Kummer $\mathrm{F}$ et al. Suture versus screw fixation of displaced tibial eminence fractures: a biomechanical comparison. Arthroscopy. 2005; 21 (10): 1172-1176. doi: 10.1016/j.arthro.2005.06.019.

6. Senekovic V, Veselko M. Anterograde arthroscopic fixation of avulsion fractures of the tibial eminence with a cannulated screw: five-year results. Arthroscopy. 2003; 19 (1): 54-61. doi: 10.1053/ jars.2003.50012. 